\title{
Structural Characteristics of Graphene Nanoclusters $\mathrm{MoS}_{2}$
}

\author{
A. M. Lohvynov \\ Sumy State University, \\ 2, R.-Korsakov Str., 40007 Sumy, Ukraine \\ e-mail: andreylogvinovsumdu@gmail.com
}

\author{
I. V. Cheshko \\ Sumy State University, \\ 2, R.-Korsakov Str., 40007 Sumy, Ukraine \\ e-mail: cheshko.iryna@gmail.com
}

\author{
M. V. Vasyukhno \\ Sumy State University, 2, \\ R.-Korsakov Str., 40007 Sumy, Ukraine
}

\begin{abstract}
The given work shows the results of the study structural-phase state of nanoclusters $\mathrm{MoS}_{2}$. The results of experimental studies have shown that the average size of $\mathrm{MoS}_{2}$ nanoclusters does not depend on the method of obtaining samples and is in the range of $L \approx 10-12 \mathrm{~nm}$. Investigation of the structure of thin layers nanoclusters $\mathrm{MoS}_{2}$ by electron method have shown that they have HCP structure with an average size lattice parameter $\bar{a}{ }_{\text {MoS2 }}=3,14 \AA$ which corresponds to the tabular value.
\end{abstract}

Index Terms-Graphene; nanoclusters; phase composition; structural state

\section{INTRODUCTION}

The $\mathrm{MoS}_{2}$-based graphene nanoclusters, due to its high thermal and electrical conductivity, transparency for light, flexibility and chemical inertness has become a promising material for use in electronics in the development of nanotransistors, spin-valve type elements, optoelectronic memory and various types of sensors [1-2] .

Therefore, research on the structural characteristics of ggraphene nanoclusters $\mathrm{MoS}_{2}$ attracts great attention of researchers. Application of $\mathrm{MoS}_{2}$ nanoclusters is relevant for the construction of miniature elements of nanoscience and microelectronics [3-5]. $\mathrm{MoS}_{2}$ is also widely used in the creation of flexible electronics, including flexible displays. In existing screens (both liquid crystal and OLED), as a transparent conductor, $\mathrm{In}_{2} \mathrm{O}_{3}$ is used, which is relatively expensive and at the same time fragile.

In this sense, the high strength and flexibility of the $\mathrm{MoS}_{2}$ nanoclusters make it an ideal candidate for the replacement of existing materials.

\section{METHOD AND TECHNIQUE OF EXPERIMENT}

To obtain thin layers of $\mathrm{MoS}_{2}$ nanoclusters, substrates were first formed of thin films of carbon $(\approx 20 \mathrm{~nm})$, which were deposited on the single crystals of salt in the medium of the «ВУП-5М» installation by a thermal method. Condensed carbon films were subsequently prepared on the support mesh substrates used in microscopic studies.

Samples were obtained from two types of $\mathrm{MoS}_{2}$ solutions of different concentrations. The first solution was obtained by Alfa Aesar (with a concentration of $12 \%$ ) and the other by Sigma (with a concentration of $25 \%$ ). The solutions were obtained by the method of banding $\mathrm{MoS}_{2}$ granules by ultrasound for 6 hours at $T=278 \mathrm{~K}$. Separation was carried out by a centrifuge at a velocity $V=21000 \mathrm{rpm}$. The average size of flakes is $\approx 100 \mathrm{~nm}$.

For condensation on a carbon substrate of $\mathrm{MoS}_{2}$ nanoclusters an ultrasonic ultrasonic disperser «УЗДН-А» in a sputtering mode is used which is used for the preparation of objects from fibrous, crystalline, powdered and other substances during electron microscopy research. In addition, the dispersant can be used to obtain suspensions and emulsions from various substances, ultrasonic cleaning of parts from mechanical contaminants, experimental studies to study the effect of ultrasound.

Electrical oscillations of $22 \mathrm{kHz}$ generated by the transistor generator of the power supply unit are converted into a piezoscircuit converter of the emitter in mechanical elastic oscillations of the corresponding frequency that affect the dispersed medium. The dispersant is made in the form of a desktop installation and is constructively a rack in which a power supply and a noise-protecting chamber are placed, walls and doors of which are reinforced with soundproof material.

A series of experiments was conducted by varying synchronization values and «УЗДН-А» intensity, distance from the nozzle to the grid, volume of the solution that was sprayed and ethanol. With the help of a dosing device, the appropriate amount of drops of $\mathrm{MoS}_{2}$ solution is applied to the «УЗДН-А» nozzle. Thus, a layer of $\mathrm{MoS}_{2}$ nanoclusters is formed on a carbon substrate.

\section{RESULTS}

The results of studies on the phase composition of thin layers of $\mathrm{MoS}_{2}$ nanoclusters (Sigma solution with a concentration of $12 \%$ ) using the «ПEM-125K» electron beam 
microscope showed that in most cases a nanostructure is formed, from which the image of the electronogram is fuzzy. The results of structural studies showed that in most cases, the formation of a structure that has a fuzzy contrast on micropictures is observed. This can be explained by the low concentration of the test solution.

The most accurate electronogram is obtained from the sample from the solution "Alfa Aesar". (Fig. 1, a). The corresponding microstructure of thin layers of $\mathrm{MoS}_{2}$ nanoclusters is shown in Fig. $1, \mathrm{~b}$ (after $T_{\mathrm{a}}=600 \mathrm{~K}$ in Fig.3). During the decoding of the diffraction pattern, the parameter of the crystalline lattice $a$ was calculated, the mean value of $\bar{a}$ $\mathrm{MoS}_{2}$, which is then compared with the table. The table value of $\mathrm{MoS}_{2}$ according to research [6] is $3.16 \AA$. The average calculated $a \mathrm{MoS}_{2}$ was $3.142 \AA$. The average value of $\bar{a} \mathrm{MoS}_{2}$ is less than the $0.018 \AA$ tablature. This difference can be explained by the fact that the table values were obtained for massive samples, and in our case a nanocluster is studied which includes a relatively small number of atoms, with a significant proportion of them on the surface of the crystalline lattice. Perhaps this causes boundary defects and distorts the crystalline lattice of a whole nanoclusters. The data of the decoding of the electron graph shown in Figure 1 (a) are given in Table 1. Thus, the decryption of the electronogram showed that the test substance completely corresponds to the hexagonal phase of $\mathrm{MoS}_{2}$.
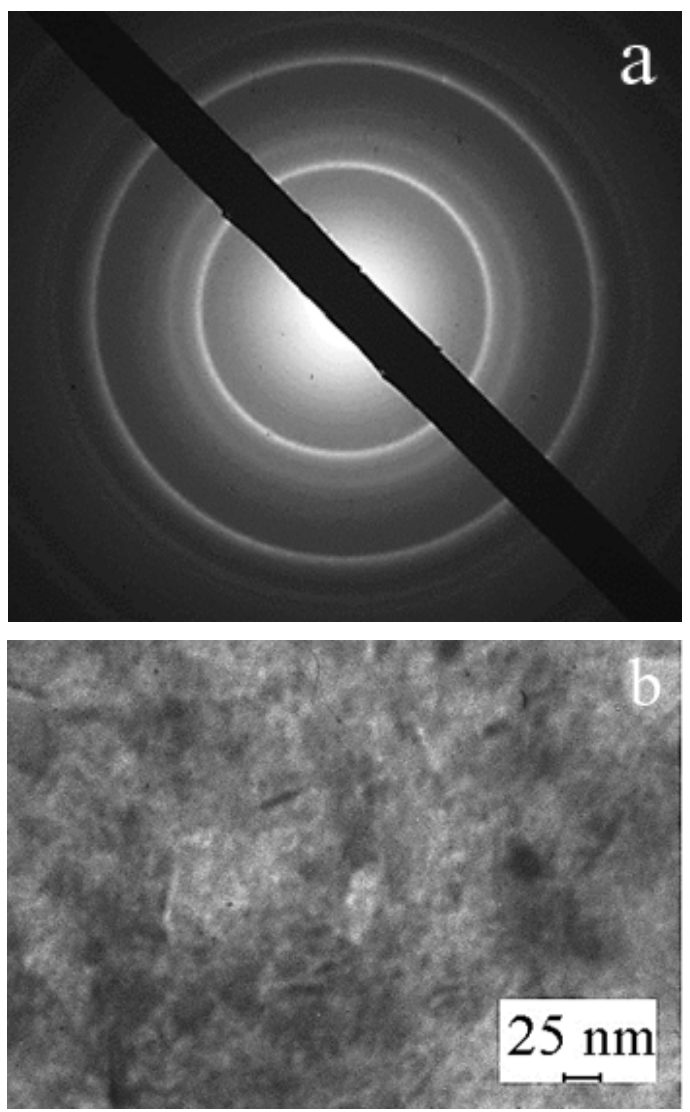

Figure 1. Electrogram (a) and corresponding microstructure (b) thin layers of $\mathrm{MoS}_{2}$ nanoclusters of the solution "Alfa Aesar"

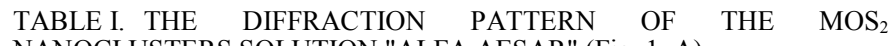
NANOCLUSTERS SOLUTION "ALFA AESAR" (Fig. 1, A)

\begin{tabular}{|c|c|c|c|c|c|c|c|}
\hline № & $\begin{array}{l}I_{\text {hkl, }} \\
\text { r.u. }\end{array}$ & $D$, r.r. & $d_{\mathrm{hkl}}, \AA$ & $h k l$ & $\begin{array}{c}d^{0}{ }_{\mathrm{hkl}}, \\
\AA\end{array}$ & $\begin{array}{c}\Delta d_{\mathrm{hkl}}, \\
\AA\end{array}$ & $a, \AA$ \\
\hline 1 & 80 & 392,5 & 2,723 & 100 & 2,737 & 0,014 & 3,144 \\
\hline 2 & 100 & 478 & 2,236 & 103 & 2,276 & 0,04 & \\
\hline 3 & 80 & 684 & 1,563 & 110 & 1,580 & 0,017 & 3,126 \\
\hline 4 & 60 & 782 & 1,367 & 200 & 1,368 & 0,001 & 3,157 \\
\hline 5 & 40 & 828 & 1,291 & 203 & 1,298 & 0,007 & \\
\hline
\end{tabular}

At lower concentrations of a solution, nanostructures with a weak contrast on micropictures are formed. We can assume that these structures have a uniform single-layered distribution of nanoclusters throughout the substrate surface.

In fig. 2 the results of study of the structural characteristics of the sample from the solution "Alfa Aesar" obtained in other conditions (reduced synchronization and intensity of the dispersant) are presented. It is evident from the microstructure that under the chosen parameters of the experiment, $\mathrm{MoS}_{2}$ nanoclusters form a dense structure. Different contrast in different areas of the micro-picture suggests that the structure formed has a layering of nanoclusters. Such a multilayer layer made it possible to investigate the phase composition of the nanosystems obtained.

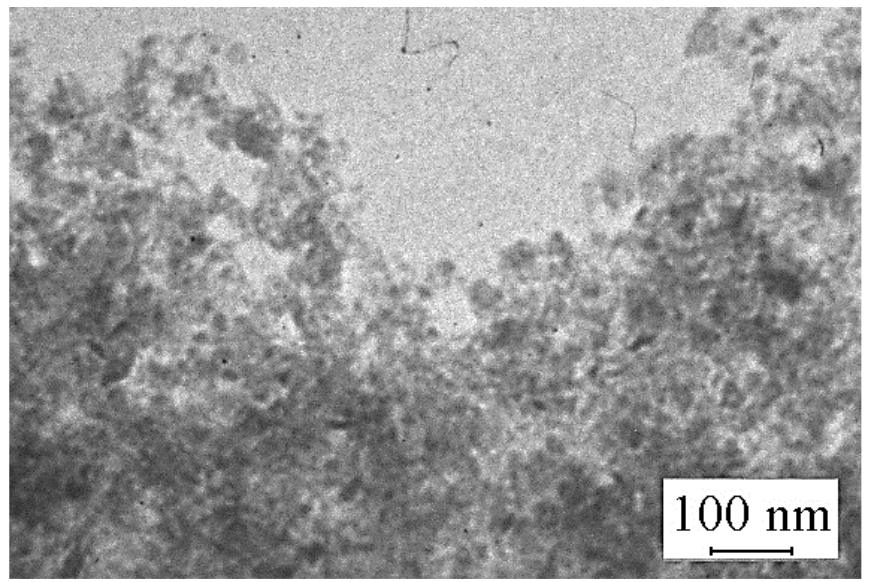

Figure 2. The structure of thin layers of $\mathrm{MoS}_{2}$ nanoclusters conglomerates from the solution of "Alfa Aesar"

The average and most likely (ML) size of the nanoclusters for the structure shown in Figure 2 was calculated. The average value of the nanostructure size is $\approx 40 \mathrm{~nm}$. The most probable value is $L_{\mathrm{ML}} \approx 14.3 \mathrm{~nm}$. The most likely size of conglomerates is $L_{\mathrm{ML}} \approx 57.1 \mathrm{~nm}$. Thus, we can conclude that this structure is a cluster of conglomerates, which in turn consist of nanoclusters.

According to the results of structural-phase studies, we can conclude that the $\mathrm{MoS}_{2}$ nanoclusters form a dense structure. Different contrast on the micro-picture suggests that the structure formed has a layering of nanoclusters, forming a conglomerate. Such a multilayer layer made it possible to investigate the phase composition of the nanosystems obtained. 
At lower concentrations of a solution, nanostructures with a relatively small contrast on microscopic images are formed.

The results of experimental studies have shown that the average size of a solution of $\mathrm{MoS}_{2}$ nanoparticles is almost independent of the method of obtaining samples. The average distance between $\mathrm{MoS}_{2}$ nanoclusters depends on the homogeneity of the sample (or stratification). The average size of conglomerates in layering nanoclusters is about $30 \mathrm{~nm}$.

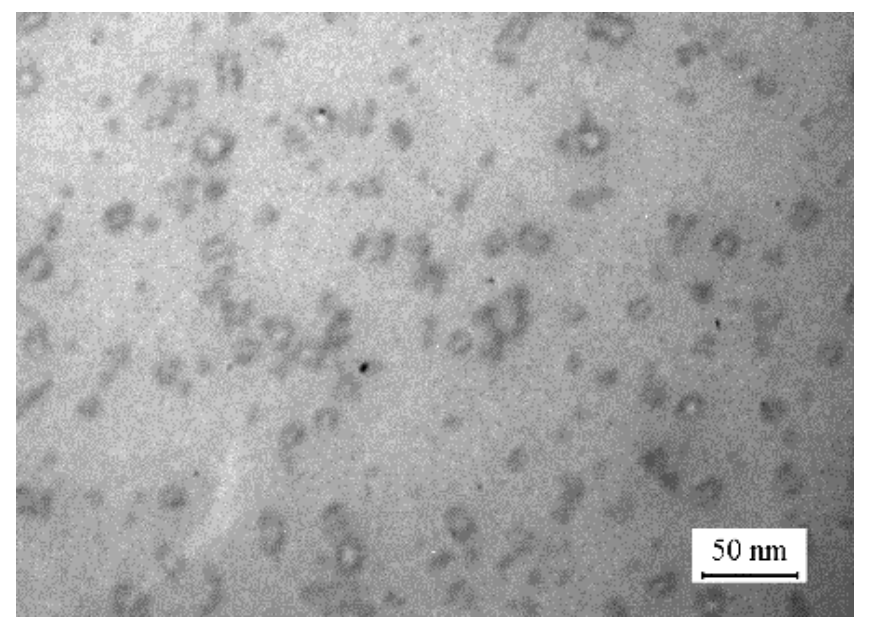

Figure 3. The structure of thin layers of $\mathrm{MoS}_{2}$ nanoclusters conglomerates from the solution of "Alfa Aesar" at the $T_{\mathrm{a}}=600 \mathrm{~K}$

Investigation of the structure of thin layers of $\mathrm{MoS}_{2}$ nanoclusters by electron-diffraction method showed that they have HCP structure with an average lattice parameter $a=3.14 \AA$, which corresponds to the table value. The study of structural characteristics showed that when spraying a solution with a concentration of $12 \%$, monolayer nanostructures with a mean nanoclusters size $L=11 \mathrm{~nm}$ are formed.

\section{CONCLUSION}

Graphene nanostroctures materials due to their properties (high thermal and electrical conductivity, transparency for light, flexibility, chemical inertness) are promising materials for use in electronics. There are prototypes of nanotransistors, elements of optoelectronic memory and various sensors based on $\mathrm{MoS}_{2}$.

To obtain thin layers of graphene $\mathrm{MoS}_{2}$ nanoclusters, ultrasonic sputtering using «УЗДН-А» using carbon films as substrates can be used.

Investigation of the structure of thin layers of $\mathrm{MoS}_{2}$ nanoclusters by electron-diffraction method showed that they have a HCP structure with an average size of the lattice parameter $\alpha=3.14 \AA$, which corresponds almost completely to the table value.

The study of structural characteristics showed that during the precipitation of the solution from the company "Sigma" monolayer nanostructures with an average size of nanoclusters $L=11 \mathrm{~nm}$ are obtained. When condensing a solution from the Alfa Aesar company, nanostructures are formed consisting of $\mathrm{MoS}_{2}$ nanoclusters conglomerates with the most probable size of nanoclusters $L_{\mathrm{ML}} \approx 14 \mathrm{~nm}$ and the most probable size of conglomerates $L_{\mathrm{ML}} \approx 57 \mathrm{~nm}$..

\section{ACKNOWLEDGMENT}

The work has been performed under the partial financial support of the Ministry of Education and Science of Ukraine (state registration number $0117 \mathrm{U} 003925$ ).

\section{REFERENCES}

[1] B. Radisavljevic, M. B. Whitwick, A. Kis, "Small-signal amplifier based on single-layer MoS 2 ,"Appl. Phys. Lett., vol. 101, pp. 4, February 2012.

[2] S. Bertolazzi, D. Krasnozhon, A. Kis, "Nonvolatile Memory Cells Based on $\mathrm{MoS}_{2} /$ Graphene Heterostructures," ACS Nano. vol. 7, pp. 3246, March 2013.

[3] Y. Yu, C. Li, Y. Liu, Scientific Reports, vol. 1, June 2013.

[4] Q. Li, M. Li, Z. Chen, C. Li, "Simple solution route to uniform $\mathrm{MoS}_{2}$ particles with randomly stacked layers," Mater. Res. Bull. vol. 39 (7-8), pp. 981, June 2004.

[5] B. Radisavljevic, A. Kis, "Mobility engineering and a metal-insulator transition in monolayer $\mathrm{MoS}_{2}$,"Nat. Mater. vol. 12, pp. 815, June 2013.

[6] K.D. Bronsema, J.L. De Boer, F. Jellinek, Allg. Chem. vol. 540, pp. 15 October 1986. 